\title{
COHOMOLOGICAL DIMENSION OF AN ABELIAN MONOID
}

\author{
CHARLES CHING-AN CHENG AND JAY SHAPIRO
}

\begin{abstract}
It is shown that the cohomological dimension of an abelian monoid is equal to that of its group reflection provided that the monoid is either finitely generated or cancellative.
\end{abstract}

Let $M$ be a monoid and let $R$ be a nonzero ring with identity. We shall denote the monoid ring of $M$ over $R$ by $R M$. The $R$-homological dimension of $M$ is defined by

$$
\operatorname{hd}_{R} M=\text { wd } R
$$

and, similarly, the $R$-cohomological dimension of $M$ is defined by

$$
\operatorname{cd}_{R} M=\operatorname{pd} R
$$

where $R$ is considered as a trivial $R M$-module (i.e. $x \cdot r=r$ for $x \in M, r \in R$ ) and where wd, pd denote weak and projective dimension respectively. Clearly hd $\mathrm{h}_{R} M<$ $\operatorname{cd}_{R} M$ and the equality holds when $R M$ is noetherian (e.g. when $R$ is noetherian and $M$ is finitely generated abelian). If there is a ring homomorphism $S \rightarrow R$ then $\operatorname{cd}_{R} M<\operatorname{cd}_{S} M$ and hd $M<\operatorname{hd}_{S} M$. In particular, $\operatorname{cd}_{R} M<\operatorname{cd} M$ and $\operatorname{hd}_{R} M<$ hd $M$ where we omit the subscript when $R=Z$. Recall that a group $M$ is said to have $R$-torsion if there exists an element of $M$ whose order is not invertible in $R$. In this case $\operatorname{cd}_{R} M=\infty$. If $M$ is an $\aleph_{n}$-generated abelian group $(n>-1)$ with no $R$-torsion, then $\operatorname{hd}_{R} M=\operatorname{rank} M$ and $\operatorname{cd}_{R} M=n+1+$ rank $M$ (see, for example, [1], [4] and [10]). In this paper we prove the following.

TheOREM A. If $M$ is an abelian monoid then $\mathrm{hd}_{R} M=\mathrm{hd}_{R} \hat{M}$.

THEOREM B. If $M$ is an abelian monoid which is either cancellative or finitely generated, then $\mathrm{cd}_{R} M=\mathrm{cd}_{R} \hat{M}$.

Here $\hat{M}$ denotes the group reflection of $M$. An example of Nico [8] shows that Theorem B does not hold for arbitrary abelian monoids.

1. Homological dimension of an abelian monoid. The group reflection $\hat{M}$ of a monoid $M$ is defined to be the image of $M$ under the left adjoint of the forgetful functor from Groups to Monoids. It is not hard to show that $\hat{M}$ is isomorphic to the quotient of the free group of $M$ by the normal subgroup generated by elements

Received by the editors September 27, 1979 and, in revised form, December 10, 1979.

1980 Mathematics Subject Classification. Primary 18G20, 20M50, 16A60, 16A62; Secondary 20M25, $13 \mathrm{DOS}$.

Key words and phrases. Cohomological dimension, monoid, group reflection. 
of form $x y z^{-1}$ where $x y=z$ and $x, y, z \in M$. There exists a monoid homomorphism $u: M \rightarrow \hat{M}$ taking $x$ to its coset. Note that each monoid homomorphism $\boldsymbol{M} \rightarrow \boldsymbol{G}$ into a group lifts (through $u$ ) to a unique group homomorphism $\hat{M} \rightarrow G$. In case $M$ is abelian the group ring $R \hat{M}$ is simply the localization of $R M$ at the central multiplicative set $M$. If, in addition, $M$ is cancellative then $\hat{M}$ is the group of quotients of $M[3, \S 1.10]$ and $u$ is injective. In case $M$ is a group we have $\hat{M}=M$.

Proof of Theorem A. Since $R \hat{M}$ is a localization of $R M$ at a central multiplicative set we have that $\operatorname{hd}_{R} M \geqslant \operatorname{hd}_{R} \hat{M}$. To prove the reverse inequality first assume that $R=K$ is commutative. Consider the exact sequence of $K M$-modules

$$
0 \rightarrow I \rightarrow K M \stackrel{\varepsilon}{\rightarrow} K \rightarrow 0
$$

where $\varepsilon$ sums coefficients. The augmentation ideal $I$ is generated by elements of the form $1-m, m \in M$. Let $p$ be a prime ideal of $K M$. If $I \nsubseteq p$ then the localization $I_{p}$ of $I$ at $p$ is simply $(K M)_{p}$ and so $K_{p}=0$. If $I \subseteq p$ then $M \subseteq K M-p$ since otherwise there exists $m \in M \cap p$ and it implies that $1=m+(1-m) \in p$. In particular, the elements of $M$ are inverted whenever we localize at prime ideals $p$ such that $K_{p} \neq 0$. Hence, localizing first at $M$ and then at $p$ is the same as localizing at $p$. Therefore,

$$
\operatorname{hd}_{K} M=\operatorname{wd}_{K M} K=\sup _{p} \operatorname{wd}_{K M_{p}} K \leqslant \operatorname{wd}_{K \hat{M}} K=\operatorname{hd}_{K} \hat{M} .
$$

In the general case let $C$ denote the center of $R$. Then $\mathrm{hd}_{R} M<\mathrm{hd}_{C} M$. On the other hand Swan [9, Proposition 3.3] proved that there exists a prime field $F$ with $\operatorname{hd}_{F} M<\operatorname{hd}_{R} M$ for any monoid $M$ such that if $M$ is a group with no $R$-torsion then it has no $F$-torsion. Suppose $\hat{M}$ has no $R$-torsion. Then it has no $C$-torsion nor $F$-torsion and, therefore, $\operatorname{hd}_{C} \boldsymbol{M}=\operatorname{hd}_{C} \hat{M}=\operatorname{rank} \hat{M}=\operatorname{hd}_{F} \hat{M}=\operatorname{hd}_{F} M$. Thus, $\operatorname{hd}_{R} M=\operatorname{hd}_{R} \hat{M}$. If $\hat{M}$ has $R$-torsion then hd ${ }_{R} \hat{M}=\infty$. Hence, using the inequality proved in the beginning, we have that $\operatorname{hd}_{R} M>\operatorname{hd}_{R} \hat{M}=\infty$.

COROLlary 1.1. If $M$ is finitely generated abelian then $\operatorname{cd}_{R} M=\operatorname{cd}_{R} \hat{M}$.

Proof. This follows from Theorem $A$ if $R$ is noetherian. Note that $\operatorname{cd}_{R} M \geqslant$ $\operatorname{cd}_{R} \hat{M}$ for any ring $R$. Clearly the equality holds if $\hat{M}$ has $R$-torsion. Suppose $\hat{M}$ has no $R$-torsion. Then $\operatorname{cd}_{R} M<\operatorname{cd} M=\operatorname{cd} \hat{M}=\operatorname{cd}_{R} \hat{M}$.

RemarK. Corollary 1.1 is no longer true if we remove the finite generation condition on $M$. For if $M=\left\{a_{i} \mid i \in \mathbf{N}\right\}$ where $a_{i} a_{j}=a_{k}$ where $k=\max (i, j)$ then $\hat{M}=1$ and cd $M>1$ (see, for example, [6] or [8]). More generally $\times{ }_{1}^{n} M$ has Z-cohomological dimension at least $n$, but its group reflection is also trivial.

2. Cohomological dimension of an abelian monoid. Throughout this section, $M$ will be an (additive) abelian cancellative monoid with group reflection $G$. Then $u$ : $M \rightarrow G$ is injective and we shall regard $M$ as a submonoid of $G$. The torsion subgroup of $G$ will be the denoted by $t G$.

LEMMA 2.1. Suppose $a, b$ are nonnegative integers, not both zero, such that $\operatorname{gcd}(a, b)=1$. Then the submonoid of the additive group $\mathbf{Z}$ of integers generated by $a$, $b$ contains all sufficiently large positive integers. 
Proof. Since $\operatorname{gcd}(a, b)=1$ there exists $s, t \in \mathbf{Z}$ with $s a+t b=1$. Note that $s$ and $t$ cannot both be nonpositive. Without loss of generality let $t>0$. Then by the Euclidean algorithm every integer larger than $a(a-1)|s|$ is of the form $a m+k$ where $m \geqslant(a-1)|s|$ and $1 \leqslant k<a$. Since $a m+k=a m+k(s a+t b)=$ $a(m-k s)+k t b$ is an element of the submonoid, the result follows.

Corollary 2.2. If $G \cong \mathbf{Z}$ then $M$ is finitely generated.

Proof. Suppose $M$ contains a positive and a negative integer. Let $s$ and $t$ be the smallest positive and largest negative integer contained in $M$ respectively. Then $t=-s$, for otherwise $s+t$ is either positive which contradicts the minimality of $s$ or it is negative which contradicts the maximality of $t$. We will prove that $s=1$ and, therefore, show that $M=G$. Every positive integer of $M$ is of the form $x=g s+r$ where $0<r<s$. Hence $r=x-g s=x+g t \in M$ contradicting the minimality of $s$ except when $r=0$. Therefore, $s$ divides all positive integers in $M$. Similarly it divides all the negative integers as well. Thus, $M$ is generated by $s$ and $-s$ and so is a subgroup of $G$ which is a contradiction unless $s=1$ since $G$ is the group reflection of $M$. Therefore, $M$ contains either only positive or only negative integers. By symmetry we assume the former. In this case there exists $a, b \in M$ with $a-b=1$. Hence, $\operatorname{gcd}(a, b)=1$. By Lemma 2.1 the submonoid generated by $a, b$ contains all sufficiently large positive integers. Therefore, $M$ is finitely generated.

LEMMA 2.3. Suppose $w \in \mathbf{Z}^{m}, m>1$, such that two of its coordinates are relatively prime. Then $\mathbf{Z}^{m} /\langle w\rangle \cong \mathbf{Z}^{m-1}$.

Proof. Clearly $\mathbf{Z}^{m} /\langle w\rangle$ has rank $m-1$ and hence it is enough to show that it is torsion-free. Suppose $y=\left(y_{i}\right) \in \mathbf{Z}^{m}$ such that $s y \in\langle w\rangle$ for some $s \in \mathbf{N}$. Then $s y=t w$ for some $t \in \mathbf{Z}$. Without loss of generality we may assume that $\operatorname{gcd}(s, t)=$ 1. Therefore, $s \mid w_{i}$ for all $i$. This implies that $s=1$ by the assumption on $w$. Hence $y=t w \in\langle w\rangle$.

Lemma 2.4. Suppose $G \nRightarrow \mathbf{Z}, G \neq 0$, and it is finitely generated with no $R$-torsion. Then there exists $x \in M$ such that $G /\langle x\rangle$ has no $R$-torsion and $\operatorname{rank} G=1+$ rank $G /\langle x\rangle$. In particular, $\operatorname{cd}_{R} G /\langle x\rangle$ is finite.

Proof. Suppose rank $G=1$. Then $G \cong \mathrm{Z} \oplus t G$ where $t G \neq 0$. There exist two elements of $M$ whose difference is $(1,0)$. Let these elements be $(a, b)$ and $(a+$ $1, b)$ where $a \in Z$ and $b \in t G$. By symmetry we may assume that $a>0$. Since $t G \neq 0$ there exists an element of $t G$ with finite order $n>1$. Since $G$ has no $R$-torsion $n$ is invertible in $R$. Using Lemma 2.1 we see that if $t$ is sufficiently large then there exists a linear combination $y \in M$ of $(a, b)$ and $(a+1, b)$ with first coordinate $n^{t}$. Set $x=k y$ where $k$ is the order of $b$. (Here we take 1 to be the order of the additive identity 0 .) Hence, the second coordinate of $x$ is zero. Therefore, $G /\langle x\rangle \cong \mathbf{Z}_{x_{1}} \oplus t G$ where $x_{1}$ denotes the first coordinate of $x$. Clearly rank $G /\langle x\rangle=0=\operatorname{rank} G-1$ and, since $x_{1}$ is invertible in $R$, we have that $G /\langle x\rangle$ has no $R$-torsion. 
Assume that rank $G=m>1$. Then $G \cong \mathbf{Z}^{m} \oplus t G$. We first show that there exists $w \in M$ with the first two coordinates relatively prime. There exists $y, y^{\prime}, z$, $z^{\prime} \in M$ with $y-y^{\prime}=(1,0, \ldots, 0)$ and $z-z^{\prime}=(0,1,0, \ldots, 0)$. Hence we may let $y=(a, b, \ldots), y^{\prime}=(a-1, b, \ldots), z=(c, d, \ldots)$ and $z^{\prime}=(c, d-$ $1, \ldots)$. If $b \neq 0$ let $w=\left(b^{2}-1\right) y+y^{\prime}=\left(b^{2} a-1, b^{3}, \ldots\right)$. If $c \neq 0$ let $w=$ $\left(c^{2}-1\right) z+z^{\prime}=\left(c^{3}, c^{2} d-1, \ldots\right)$. Otherwise $b=0=c$. If $d=0$ then we take $w=z^{\prime}$. If $d \neq 0$ then we take $w=\left(d^{2}-1\right) y+y^{\prime}+z=\left(d^{2} a-1, d, \ldots\right)$. Now set $x=k w$ where $k$ is the order of the last coordinate of $w$. (Here we take 1 to be the order of 0 .) Let $w^{\prime}$ and $x^{\prime}$ denote the images of $w$ and $x$ respectively under the projection of $G$ onto $Z^{m}$. Since the last coordinate of $x$ is zero we have $G /\langle x\rangle=$ $\left(\mathbf{Z}^{m} \oplus t G\right) /\langle x\rangle \simeq \mathbf{Z}^{m} /\left\langle x^{\prime}\right\rangle \oplus t G, \quad \mathbf{Z}^{m} /\left\langle x^{\prime}\right\rangle \cong \mathbf{Z}^{m} /\left\langle w^{\prime}\right\rangle \oplus\left\langle w^{\prime}\right\rangle /\left\langle x^{\prime}\right\rangle$. Note $\mathbf{Z}^{m} /\left\langle w^{\prime}\right\rangle \cong \mathbf{Z}^{m-1}$ by Lemma 2.3 and $\left\langle w^{\prime}\right\rangle /\left\langle x^{\prime}\right\rangle \cong \mathbf{Z}_{k}$. In any case rank $G=1+$ rank $G /\langle x\rangle$ and $G /\langle x\rangle$ has no $R$-torsion since $k$ is invertible in $R$ and $G$ has no $R$-torsion.

Lemma 2.5 (Kaplansky [5, Part III, Theorem 3]). Suppose $R$ is a ring with identity and $x$ is central in $R$. If $x$ is not a zero divisor and $R^{*}=R /(x)$ then for each $R^{*}$-module $A \neq 0$ with $\operatorname{pd}_{R^{*}} A=n$ we have that $\operatorname{pd}_{R} A=1+n$.

Proposition 2.6. If $G$ is finitely generated then $\operatorname{cd}_{R} M=\mathrm{cd}_{R} G$.

Proof. Since $\operatorname{cd}_{R} M>\operatorname{cd}_{R} G$ always, we need only to consider the case when $\mathrm{cd}_{R} G$ is finite. Hence, $G$ has no $R$-torsion and finite rank. If rank $G=0$ then $G=t G$ and so $M$, as a submonoid of a torsion group, is a group and hence is equal to $G$. In particular, $\operatorname{cd}_{R} M=\operatorname{cd}_{R} G$. Assume rank $G>0$. If $G \simeq \mathrm{Z}$ then this follows from Corollaries 1.1 and 2.2. Therefore, we may assume $G \approx \mathbf{Z}$. By Lemma 2.4 there exists $x \in M$ such that $\operatorname{rank} G=1+\operatorname{rank} G /\langle x\rangle$ and $\operatorname{cd}_{R} G /\langle x\rangle<$ $\infty$. Let $\bar{M}$ denote the image of $M$ under the canonical map $G \rightarrow G /\langle x\rangle=\bar{G}$. It is easy to see that $\bar{G}$ is the group reflection of $\bar{M}$. Hence, by the induction assumption, we have $\operatorname{cd}_{R} \bar{M}=\operatorname{cd}_{R} \bar{G}$. On the other hand it is not difficult to show that $R \bar{M} \cong R M /\langle x-1\rangle$ and $R \bar{G} \cong R G /\langle x-1\rangle$. Since $x-1 \in R M$ is not a zero divisor and is central in $R M$ we obtain from Lemma 2.5 that $\operatorname{cd}_{R} M=\operatorname{pd}_{R M} R=$ $1+\operatorname{pd}_{R \bar{M}} R=1+\operatorname{cd}_{R} \bar{M}$ and, $\operatorname{cd}_{R} G=1+\operatorname{cd}_{R} \bar{G}$. Thus $\operatorname{cd}_{R} M=\operatorname{cd}_{R} G$.

REMARK. It should be pointed out that $M$ may not be finitely generated even when $G$ is finitely generated. For example let $G=\mathbf{Z}^{2}$ and $M=\langle\{(m, 1) \mid m \in \mathbf{N}\}\rangle$. Thus Proposition 2.6 is significantly different from Corollary 1.1 .

Proposition 2.7. Suppose $M=\lim _{I} M_{i}$ where $I$ is a directed set of cardinality $\boldsymbol{x}_{n}$ $(n>-1)$ and $M_{i}$ 's are monoids (not necessarily abelian). Then

$$
\operatorname{cd}_{R} M<n+1+\sup _{I} \operatorname{cd}_{R} M_{i} \text {. }
$$

Proof. This follows from Theorem 37.1 of Mitchell [7] together with the observation that $(M, R)$ is the direct limit of $\left(M_{i}, R\right)$ in Addfun $A b$.

Proof of Theorem B. By Corollary 1.1 we may assume that $M$ is cancellative. Since $\operatorname{cd}_{R} M \geqslant \operatorname{cd}_{R} G$ we may assume that $\operatorname{cd}_{R} G$ is finite. Hence, $G$ has no $R$-torsion and rank $G=m<\infty$. If $G$ is finitely generated then the result is simply Proposition 2.6. Assume that $G$ is $\boldsymbol{x}_{n}$-generated with $n>0$. Then $M$ is also 
$\aleph_{n}$-generated, and so $M=\lim _{I} M_{i}$ where $M_{i}$ 's are finitely generated submonoids of $M$ and $|I|=\aleph_{n}$. By Proposition 2.7, $\operatorname{cd}_{R} M<n+1+\sup _{R} \operatorname{cd}_{i}=n+1+$ sup $\operatorname{cd}_{R} \hat{M}_{i}<n+1+\operatorname{rank} G=\operatorname{cd}_{R} G$, where $\hat{M}_{i}$ is the group reflection of $M_{i}$.

ACKNOWLEDGEMENT. The first named author would like to thank Oakland University for a research grant which enabled him to spend the summer of 1978 at the Institute for Advanced Study where a portion of this paper was written. He is also indebted to the Institute for the hospitality shown. Finally, the authors would like to thank the referee for pointing out [1] which led to more complete results.

\section{REFERENCES}

1. S. Balcerzyk, The global dimension of the group rings of abelian groups. III, Fund. Math. 67 (1970), 241-250.

2. H. Cartan and S. Eilenberg, Homological algebra, Princeton Univ. Press, Princeton, N. J., 1956.

3. A. H. Clifford and G. B. Preston, The algebraic theory of semigroups. I, Math. Surveys, No. 7, Amer. Math. Soc., Providence, R.I., 1961; reprinted 1977.

4. G. L. Feldman, On the homological dimension of group algebras of solvable groups, Math. USSR-Izv. 5 (1971), 1231-1244.

5. I. Kaplansky, Fields and rings, Univ. of Chicago Press, Chicago, Ill., 1969.

6. O. A. Laudal, Note on the projective limit on small categories, Proc. Amer. Math. Soc. 33 (1972), 307-309.

7. B. Mitchell, Rings with several objects, Advances in Math. 8 (1972), 1-161.

8. W. R. Nico, A counter-example in the cohomology of monoids, Semigroup Forum 4 (1972), 93-94.

9. R. G. Swan, Groups of cohomological dimension one, J. Algebra 12 (1969), 585-610.

10. K. Varadarajan, Dimension, category and $K(\pi, n)$ spaces, J. Math. Mech. 10 (1961), 755-771.

Department of Mathematical Sciences, OAKIANd University, Rochester, Michigan 48063

Department of Mathematics, Wayne State University, Detrort, Michigan 48202 